\title{
Characterizing the face in facioscapulohumeral muscular dystrophy
}

\author{
T. G. J. Loonen ${ }^{1,2} \oplus$. C. G. C. Horlings ${ }^{1}$ - S. C. C. Vincenten ${ }^{1}$ - C. H. G. Beurskens ${ }^{3} \cdot$ S. Knuijt ${ }^{4}$ - G. W. A. M. Padberg ${ }^{1}$. \\ J. M. Statland ${ }^{5} \cdot$ N. C. Voermans ${ }^{1} \cdot$ T. J. J. Maal $^{2}$ - B. G. M. van Engelen ${ }^{1} \cdot$ K. Mul $^{1}$
}

Received: 14 September 2020 / Revised: 14 October 2020 / Accepted: 20 October 2020 / Published online: 28 October 2020 (c) The Author(s) 2020

\begin{abstract}
Objective To evaluate facial weakness in patients with FSHD to better define clinical signs, and pilot a facial weakness severity score.

Methods 87 FSHD patients and 55 controls were video recorded while performing seven facial tasks. The videos were assessed by three independent examiners to compile an overview of signs of facial weakness. Next, videos were semi-quantitatively assessed using a newly developed 4-point facial weakness score (FWS). This score was evaluated and correlated to other FSHD disease characteristics.

Results Patients had lower scores on the total FWS than controls (mean score 43 \pm 28 , range 4-118, vs 14 \pm 9 , range 0-35, $p<0.001$ ) and on all seven individual facial tasks (all $p<0.001$ ). $54 \%$ of patients had FWS scores outside the range of controls. Patients had more asymmetry between the left and right side of the face than controls. About $10 \%$ of the patients had very mild facial weakness. These were mostly males (89\%) with longer D4Z4 repeat sizes of 7-9 units. More severe facial weakness correlated to more severe overall disease severity and shorter D4Z4 repeat size, but not to disease duration. Interobserver agreement for the FWS between three raters was low with a Fleiss Kappa of 0.437.

Conclusion This study provides an overview of the clinical spectrum of facial weakness and its relation to other disease characteristics. The 4-point scale we introduced to grade the severity of facial weakness enables correlation of facial weakness to disease characteristics, but is not suited as clinical outcome measure for longitudinal studies.
\end{abstract}

Keywords Facioscapulohumeral muscular dystrophy $\cdot$ Facial weakness $\cdot$ Outcome measures

\author{
K. Mul \\ karlien.mul@radboudumc.nl \\ T. G. J. Loonen \\ tom.loonen@radboudumc.nl \\ C. G. C. Horlings \\ Corinne.Horlings@radboudumc.nl \\ S. C. C. Vincenten \\ Sanne.Vincenten@ radboudumc.nl \\ C. H. G. Beurskens \\ carien.beurskens@gmail.com \\ S. Knuijt \\ Simone.Knuijt@radboudumc.nl \\ G. W. A. M. Padberg \\ georgepadberg@gmail.com \\ J. M. Statland \\ JStatland@kumc.edu \\ N. C. Voermans \\ Nicol.Voermans@radboudumc.nl
}

T. J. J. Maal

Thomas.Maal@radboudumc.nl

B. G. M. van Engelen

Baziel.vanEngelen@radboudumc.nl

1 Department of Neurology, Donders Institute for Brain, Cognition and Behaviour, Radboud University Medical Center, PO Box 9101, 6500 HB Nijmegen, The Netherlands

2 Radboudumc 3D-Lab, Department of Oral and Maxillofacial Surgery, Radboud University Nijmegen Medical Centre, Nijmegen, The Netherlands

3 Department of Orthopedics, Section of Physical Therapy, Radboud University Medical Center, Nijmegen, The Netherlands

4 Department of Rehabilitation, Donders Institute for Brain, Cognition and Behaviour, Radboud University Medical Center, Nijmegen, The Netherlands

5 Department of Neurology, University of Kansas Medical Center, Kansas City, KS, USA 


\section{Introduction}

Facioscapulohumeral muscular dystrophy (FSHD) is a progressive inherited muscle disorder. A highly characteristic sign of FSHD is facial weakness that may vary between patients from minimal asymmetry to myopathic facies $[1,2]$. The circular muscles around the eyes and mouth (orbicularis oculi and orbicularis oris, respectively) and the muscle that raises the corners of the mouth (zygomaticus major) are said to be commonly affected $[1,3]$. A recent study suggests that other facial muscles, like for example the $\mathrm{m}$. buccinator, may be affected in FSHD as well [2]. The facial weakness results in functional impairments such as difficulties in eating, drinking, speaking, and ocular problems, as well as in a reduced ability for facial expressions, hindering non-verbal communication.

In spite of its clinical relevance, facial weakness is a neglected feature in the consulting room and in research on FSHD [4]. Much is known about the specific pattern of involvement of the limb muscles, but studies describing the clinical characteristics of facial weakness in FSHD are scarce. Little is known about the prevalence and (variability in) the severity of facial weakness, its progression over time, relation to other disease characteristics, and the consequences of facial weakness for the patients.

This is probably due to the fact that we are lacking the clinical approach or tools to structurally assess facial weakness and measure changes, and the effect of those changes, over time.

To approach the current gap in the availability of tools for tracking facial weakness we video recorded faces of FSHD patients and controls to better define clinical signs of facial weakness to accommodate optimal examination. Next, we pilot a newly developed severity score for facial weakness to enable comparison between patients, correlations to other disease characteristics, and potentially track changes over time.

\section{Methods}

\section{Patients}

We included genetically confirmed FSHD patients aged 18 years or older from a large cross-sectional cohort study on FSHD performed at the Neurology department of the Radboud University Medical Center (Nijmegen, the Netherlands) from 2014 to 2015 (FSHD-FOCUS study) [5]. Study visit companions were invited to participate in the non-FSHD control group.

\section{Standard protocol approvals, registrations and patient consents}

This study was conducted according to the principles of the Declaration of Helsinki (version October 2013) and in accordance with the Medical Research Involving Human Subjects Act (WMO). The study protocol was approved by the regional medical ethics committee (CMO Arnhem-Nijmegen). Prior to inclusion, all participants signed informed consent and if applicable a consent-to-disclose form.

\section{Data acquisition}

All participants were video recorded while performing clinical exam procedures to elicit facial weakness. Seven different facial tasks were performed in random order: closing the eyes gently, closing the eyes firmly, raising the eyebrows, frowning, pursing the lips, showing the teeth and puffing of the cheeks (Fig. 1). All tasks started with a relaxed state of facial muscles and ended with the maximal movement possible. Each movement was performed three times in sequence during a session, resulting in twenty-one (seven times three) tasks per video (one video per participant).

The videos were recorded with a Canon PowerShot SX280 HS camera, which was positioned $70 \mathrm{~cm}$ in front of the participant. The camera was set to record at a resolution of $1920 \times 1680$ at 60 frames per second.

\section{Assessment of videos}

The videos of all patients were independently assessed by three researchers (authors TL, SV and KM). Per video the researchers listed all clinical signs they noticed that could indicate facial weakness. Their assessments were combined to compile an overview of all signs noted to better define clinical signs of facial weakness.

Next, videos were semi-quantitatively assessed using a newly developed 4-point score for facial weakness, ranging from normal movements without effort (0) to the inability to initiate or perform a movement (3) (Table 1). Members of the study team with extensive experience with FSHD selected the set of facial movements likely to show changes in FSHD patients. Next, four different response options were formulated based on observations of clinical features in the videos. The assessments were performed independently by three experts in facial weakness from different disciplines [a mime therapist (CB), a neurologist specialized in FSHD (GP) and a speech language pathologist with special expertise in neuromuscular diseases (SK)]. All videos were cropped to an extend of which they only showed the 
Fig. 1 Face in rest and facial tasks in one of the controls. a Resting position; b closing the eyes gently; c closing the eyes firmly; $\mathbf{d}$ raising the eyebrows; e frowning; f pursing the lips; $\mathbf{g}$ showing the teeth and $\mathbf{h}$ puffing of the cheeks

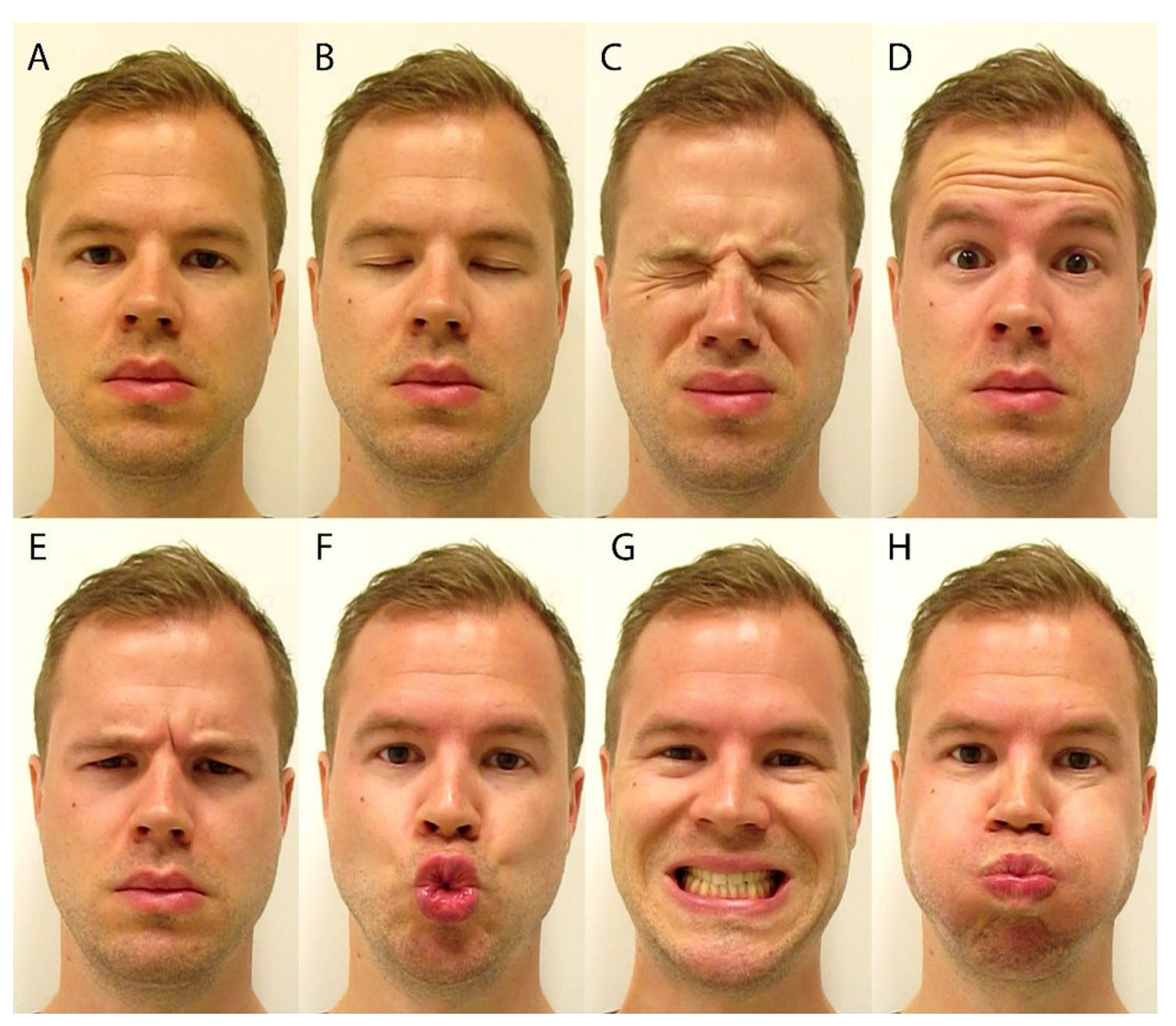

\begin{tabular}{lll}
\hline Score & Mouth and forehead & Eye closure \\
\hline 0 & Complete movement possible & Complete eye closure \\
1 & Near complete movement possible & Small rim of eyelashes visible on closure \\
2 & Limited movement possible & Eyelashes mostly visible on closure \\
3 & Impossible to initiate movement & Incomplete eye closure \\
\hline
\end{tabular}

Table 1 Instructions on the scoring sheet for the facial weakness score 


\section{Statistical analysis}

Descriptive statistics were calculated for all participants. Mean and standard deviation are reported unless stated otherwise. The Fleiss Kappa value was calculated for the reliability of agreement between the three observers. Spearmen's rho correlation was used for correlations between ordinal scores and Pearson correlation for linear variables. Statistical analysis were performed using SPSS version 22, version 3.5.3.

\section{Results}

\section{Patient characteristics}

Eighty-seven FSHD patients and fifty-five controls were included in this study. Baseline characteristics are presented in Table 2. Two of the eighty-seven FSHD patients received nocturnal non-invasive ventilation.

\section{Overview of signs of facial weakness}

An overview of all clinical signs of facial weakness that were noted in the video's is given below to aid in the clinical examination of patients. Examples of moderate and severe weakness are displayed in Fig. 2.

\section{Upper part of the face}

In resting position, subtle weakness of the muscles around the eyes can be noted by a difference in palpebral fissure height, red conjunctiva when the eyes are dry, and in more severe cases hanging of the lower eyelid. Especially older patients may have less wrinkles around the eyes and on the forehead compared to controls of the same age.
When patients are asked to close their eyes (gently) a Bell's phenomenon may occur, although this also occurred in some of the controls. In patients with more severe facial weakness there is an inability to fully close the eyes. By having the patient close the eyes forcefully more subtle weakness can be detected, especially a 'signe de cils' - an inability to bury the eyelashes completely when attempting to close the eyes tightly. To compensate for the weakness around their eyes, FSHD patients are more likely to move their mouth when asked to close their eyes forcefully. Additionally, their foreheads show less wrinkling, as was also the case when asking the patients to raise their eyebrows or to frown.

In rest, the position of the eyebrows is asymmetrical in part of the patients. This becomes more evident when the patient raises the eyebrows or frowns. In contrast, in controls the asymmetry tends to resolve when the patient moves the eyebrows. The range of motion of the eyebrows is often smaller in FSHD patients compared to controls.

Although we did not specifically test the movement of the eyes, we did not see signs suggestive of involvement of extraocular muscles. No ptosis was seen.

\section{Lower part of the face}

In part of the patients the mouth is asymmetrical in rest which can be noticed by a difference in position of the corners of the mouth, or in more severe cases the philtrum is skewed to one side. Severe weakness of the muscles around the mouth can cause the lower lip to drop. Asymmetry becomes more pronounced when a patient performs a task like pursing the lips or showing the teeth (raising the corners of the mouth). When performing these tasks some patients are able to only partly execute the movement, or are able to complete the movement but not to maintain the final position.
Table 2 Demographic data of study population

\begin{tabular}{llll}
\hline & Patients $n=87$ & Controls $n=55$ & $p$ value \\
\hline Male $(n,(\%))$ & $51(58 \%)$ & $27(47 \%)$ & $>0.05$ \\
Age in years (mean \pm SD [range]) & $56.0 \pm 15.4[23-86]$ & $50.9 \pm 16.3[21-87]$ & $>0.05$ \\
FSHD type 1 & $n=80$ & $\mathrm{n} / \mathrm{a}$ & \\
FSHD type 2 & $n=5$ & & \\
FSHD type 1+2 & $n=2$ & $\mathrm{n} / \mathrm{a}$ & \\
D4Z4 repeat array size in units & $n=15$ & & \\
2-4 & $n=27$ & & \\
5-6 & $n=40$ & & \\
7-9 & $n=5$ & $14 \pm 9[0-35]$ \\
FSHD type 2 & & $\mathrm{n} / \mathrm{a}$ \\
Facial weakness score (mean \pm SD [range]) & $43 \pm 28[4-118]$ & $\mathrm{n} / \mathrm{a}$ & \\
FSHD evaluation score (mean \pm SD [range]) & $6.9 \pm 4.2[0-15]$ & $5.5 \pm 2.8[0-10]$ & \\
Ricci score (mean \pm SD [range]) & & \\
\hline
\end{tabular}

n/a not applicable 


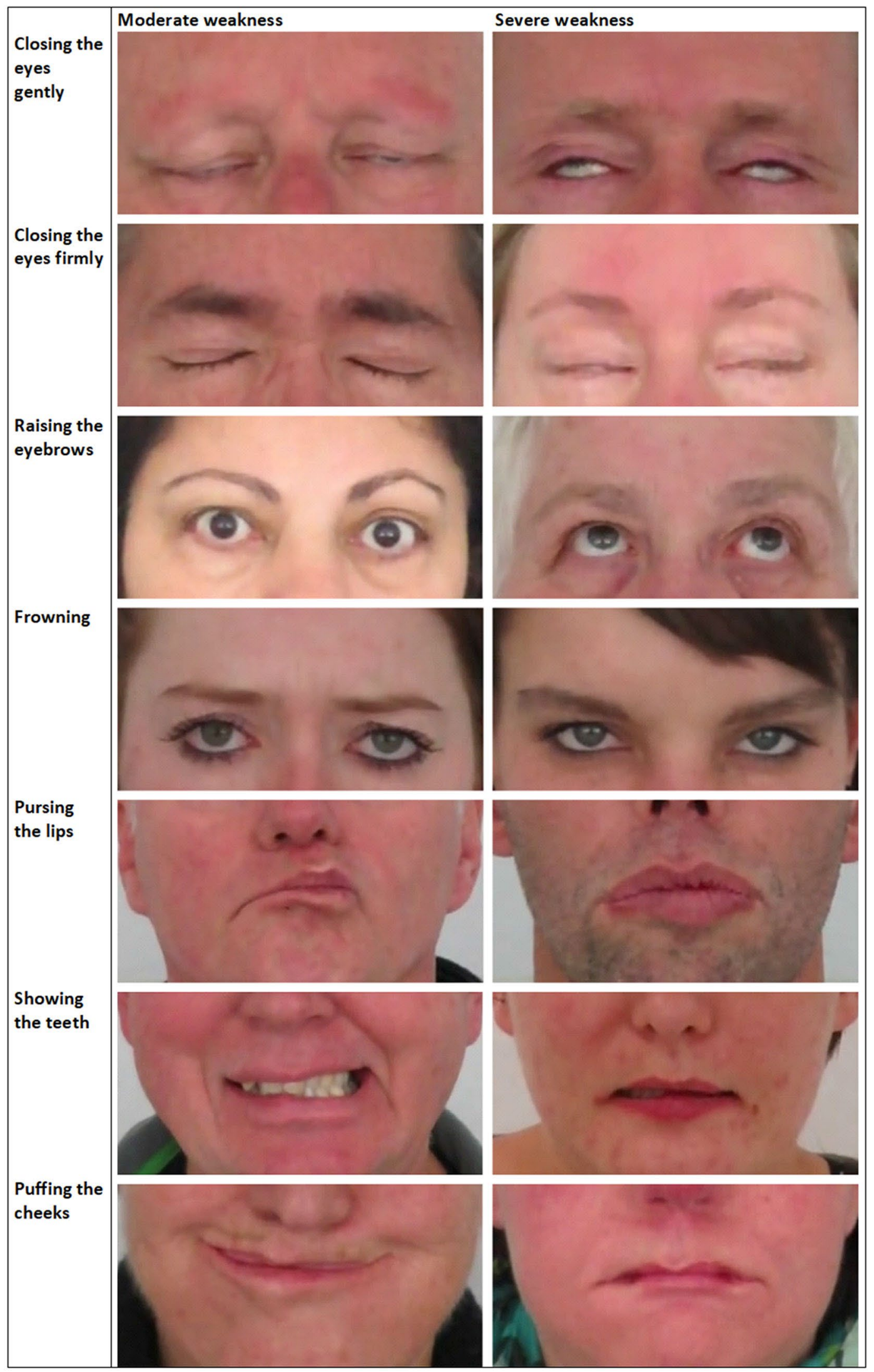

Fig. 2 Examples of moderate and severe facial weakness per task (image taken of maximal movement possible) 


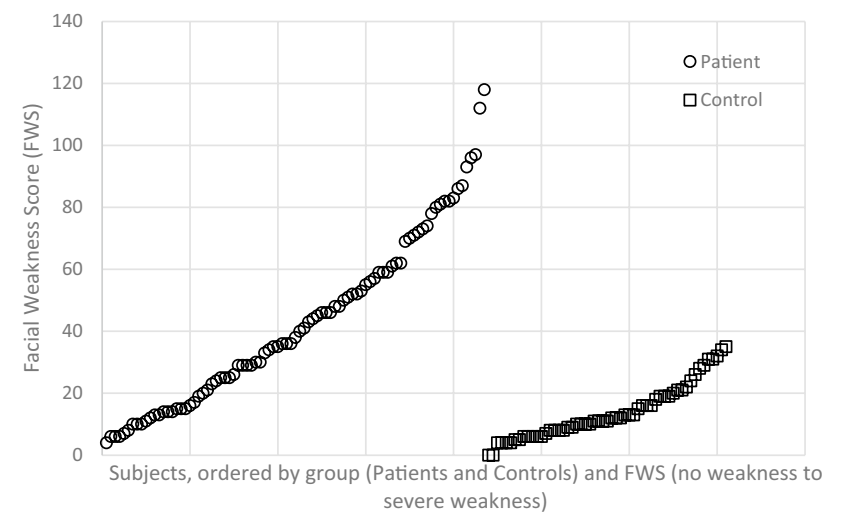

Fig. 3 Facial weakness score per individual participant ranked per group from lowest to highest score

When patients attempt to smile, a so called 'transverse smile' is often seen. Due to the inability to raise the corners of the mouth, the mouth moves horizontally which may look like a grin. In cases of very severe facial weakness patients are not able to raise the corners of the mouth at all and instead move their lower yaw forward when asked to show their teeth.

\section{Facial weakness score}

In patients the total score on the FWS ranged from 4 to 118 , compared to a range between 0 and 35 in the controls. Although there was overlap between the scores of the patients and the controls, $54 \%$ of the patients had scores above the upper limit of the control scores (Fig. 3). Patients had lower scores than controls on the total FWS (mean score $43 \pm 28$ vs $14 \pm 9$ respectively, $p<0.001$ ) as well as on all the seven individual facial tasks (all $p<0.001$ ). There was no difference in the severity of the weakness between the upper and lower part of the face (no difference in mean scores for the tasks concerning weakness in the upper half versus the lower half of the face).

Males and females with FSHD had the same degree of facial weakness (mean total FWS $38 \pm 25$ vs $50 \pm 30$ respectively, $p>0.05)$.

Patients had more asymmetry between the left and the right side of the face than controls (mean absolute difference between scores for left and right side of the face $5.1 \pm 3.6 \mathrm{vs}$ $1.7 \pm 1.8, p<0.001)$. In patients, there was no preference for either right- or left-sided facial weakness.

Interobserver agreement for the total FWS score between the three experts was generally low with a Fleiss Kappa for the total FWS score of 0.437. Although the Fleiss kappa values varied somewhat between the various facial movement tasks, interobserver agreement was poor to moderate for all tasks (all Fleiss kappa $\leq 0.52$ ). Collapsing the response categories from four to three (0-1-2-3 to 0-1-1-2) improved

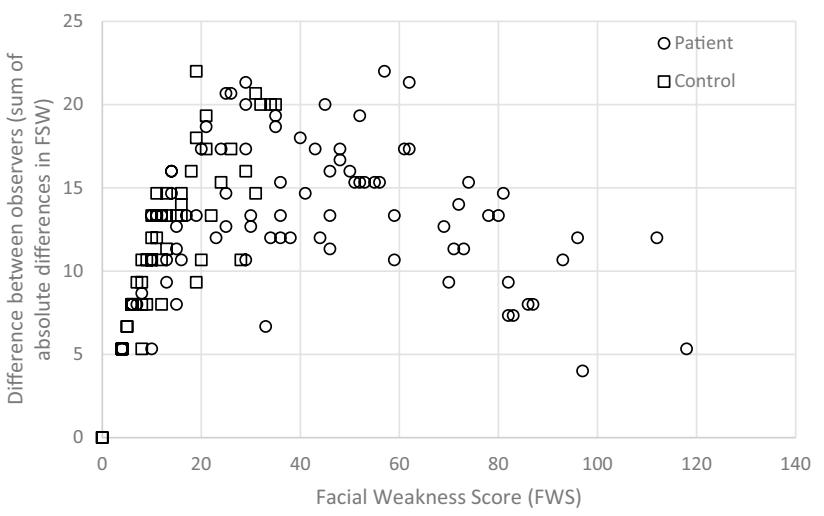

Fig. 4 Sum of absolute differences in FWS between observers per individual. For individuals with moderate weakness the variability between observers is higher than for individuals with mild and severe weakness

interobserver agreement to 0.498. Disagreement between the three experts was most pronounced with intermediate severity of facial weakness (Fig. 4).

\section{Correlation between FWS and FSHD disease characteristics}

Facial weakness was more severe when overall disease severity was more severe on both the FSHD evaluation score and Ricci score $(\rho 0.561, p<0.001$ and $\rho 0.465, p<0.001$ respectively) and D4Z4 repeat size was shorter $(\rho=-0.507$, $p<0.01$ ).

Although patients with early onset FSHD (shoulder girdle weakness $\leq 10$ years of age, $n=6$ ) had more severe facial weakness than classical onset patients (mean FWS of $91 \pm 17$ vs $39 \pm 25, p<0.001$ ), there was no correlation between disease duration and the degree of facial weakness $(r=0.204, p=0.085)$. A weak correlation was found between the degree of facial weakness and age in the patients $(\rho=0.280, p=0.009)$, but not in the control group $(\rho=-0.244, p=0.072)$.

\section{Facial sparing phenotype}

We identified nine out of 87 patients (eight males, age range 41-73 years) that fulfilled our criteria for a facial sparing phenotype. Their FWS results ranged from 7 to 15 . All of these patients had a D4Z4 repeat array size of five to nine units, except for one who was an FSHD2 patient.

In six of these nine patients the experts observed clear, though mild, signs of facial weakness (all males, age range 48-73 years) (Fig. 5). All of these patients had notable asymmetry with movements of the mouth, i.e. single sided weakness of the muscles around the mouth. Three of them 

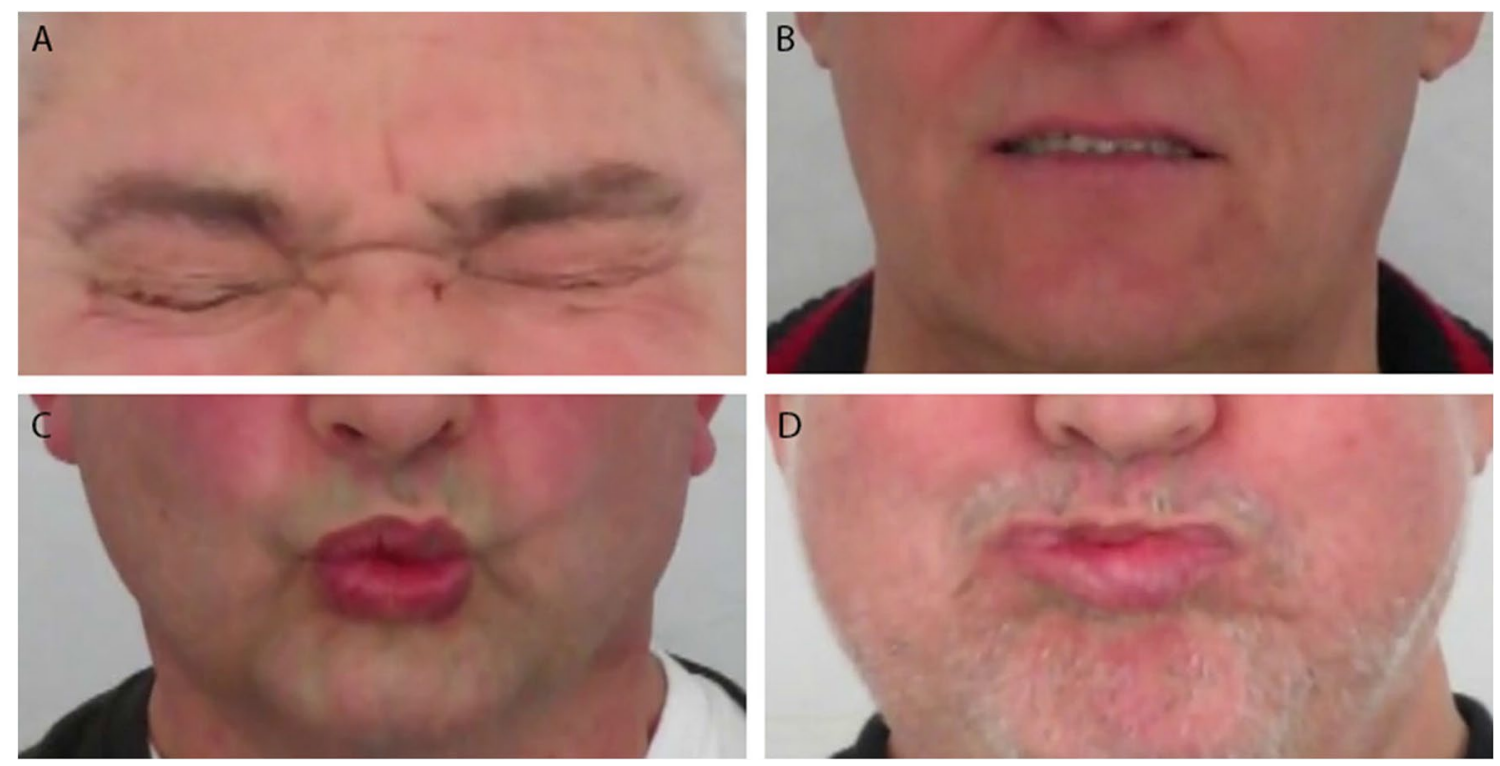

Fig. 5 Examples of subtle signs of facial weakness. a Signe de cils right eye (inability to bury the eyelashes completely when attempting to close the eyes tightly). b Asymmetry in raising the corners of the mouth with left corner being raised less high. c Asymmetry when

had signe de cils. One patient had trouble raising his left eyebrow.

The other three patients had such minimal signs of facial weakness that they could easily go unnoticed upon examination ( 2 males, ages 41, 47 and 62 years). One male patient only had rightsided signe de cils. The other male patient had signe de cils and although he was able to completely raise the corners of his mouth, he could not sustain the maximum position for more than a second with the left corner of his mouth. The female patient had asymmetrical movements of the corners of the mouth (right side performed suboptimal) when showing the teeth and blowing the cheeks.

\section{Discussion}

In this study we systematically assessed facial weakness in FSHD in a large group of patients comprising the entire clinical spectrum.

Fifty-four percent of the FSHD patients had an FWS above the upper limit of the FWS of the control group. This indicates that the group of patients with moderate to severe weakness can adequately be distinguished from non-FSHD controls, but in the group of patients with milder weakness assessing facial weakness becomes more complex. Only two of all the participants had a completely normal score on the FWS. This finding shows that mild signs of facial weakness are not necessarily specific for FSHD, but can be seen in the general population. pouting the lips due to right sided weakness of the muscles around the mouth. d Difficulty puffing the cheeks. Notice the asymmetry and the lips being sealed horizontally

One of the signs of facial weakness that is more specific for FSHD is left-right asymmetry, as asymmetry was more common in patients than controls and patients with very mild facial weakness mostly had single sided weakness. More research is required to define other signs of facial weakness that can help to reliably discriminate between patients and controls.

Some of the controls had relatively high scores (up to 35) on the FWS. These scores are probably the result of various factors influencing the score. First, despite verbal instructions some of the controls performed the movements technically poorly or not with maximum effort, which did not seem to be caused by muscle weakness. Second, the experts did not know if they were watching a video of a patient or a non-FSHD volunteer, which poses a risk of experimenter's bias when scoring the tasks: a tendency of rating values to drift towards what is expected by the rater, i.e. a tendency to assign higher scores when they suspected that the participant had FSHD. This is based on the idea that experienced researchers evaluate the presence of FSHD in a holistic view of the face and movement of the face rather than based on individual parts and exercises. Finally, no information was collected regarding comorbidities that could have an effect on facial muscle function or facial expression in the control group.

Approximately $10 \%$ of the patients had very mild weakness (within the bottom 75\% FWS range of the control group). This is line with previous studies reporting a facial sparing phenotype in approximately $15 \%$ of cases $[3,8]$. 
Our study shows, however, that the definition of 'facial sparing FSHD' remains challenging. All patients in the study that could potentially be classified as having a facial sparing phenotype did have mild facial weakness, but this weakness fell within the range of what is seen in the control population. We identified multiple patients with very subtle signs of facial weakness, that were not recognized by the patient and could easily be overlooked by an examiner. As such, the term 'facial sparing' should be used with caution in the hands of an observer who is not thoroughly familiar with FSHD.

In accordance with the literature, very mild facial weakness was observed in patients with longer repeat array sizes of 7-10 D4Z4 units [8-13], which suits the finding that the D4Z4 repeat array has a stronger influence of the degree of facial weakness than on the upper and lower extremity muscle involvement [5].

Although there was no difference in mean FWS between males and females, we found that $89 \%$ of the patients with a facial sparing phenotype was male. This is in concordance with other studies reporting $67-100 \%$ of patients being male in series ( $\geq 3$ patients) of patients with a facial sparing phenotype [8-10, 13-17]. Other sex differences have been observed in FSHD, such as a higher proportion of women among asymptomatic gene carriers and a higher frequency of STIR positive lesions on muscle MRI in males, but these are all still pathophysiologically unexplained.

We observed no correlation between the severity of the facial weakness and the duration of the disease, although there was a weak positive correlation between facial weakness and age in the patients. This suggests that facial weakness in FSHD shows only little progression over time compared to the limb muscles or that progression only occurs during a short time interval in life. Longitudinal studies should be performed to provide information on progression of facial weakness. However, to enable longitudinal studies, both in the setting of a clinical trial or natural history study, an adequate outcome measure for facial weakness is required.

The semi-quantitative 'facial weakness score' presented here allowed us to grade the severity of facial weakness and subsequently explore the relation between facial weakness and other disease characteristics. The score showed a poor agreement between different observers and a range of scores in the control group. Therefore, the proposed score in its current form serves to make progress in this research field, but cannot be used as a clinical outcome measure.

Different explanations for the low interrater agreement are possible. First, the experts who assigned the scores received limited training to perform the scoring and all three had a different background, which may result in different interpretations of findings on the facial tasks. Second, the risk of experimenter's bias as mentioned above could result in different scores between observers, depending on whether they believed they were watching a video of a patient or a control. Third, properties of the score itself can contribute to a lower reliability. The number of response options may have been too high to be able to discriminate consistently between different answering options, although reducing the number of response categories only mildly improvement the interrater agreement, or the phrasing of the response options may have been unclear. Although unlikely to resolve the low interrater agreement, spontaneous mimical movements and assessment of the face at rest would be of interest to add in future work on this topic. Finally, the complexity of the face and its movements makes it challenging to develop a (semi) quantitative scoring system, which is also illustrated by the large intra- and interobserver variability when applying different scoring systems in studies focused on facial nerve dysfunction [18]. This complexity means that a scoring system must contain many parameters to provide a reliable measure. Finding an easy to implement measure for a complex problem seems a perfect challenge for a computercontrolled solution like artificial intelligence, thus more research is needed to investigate the feasibility of such a solution. Future development of a computer-controlled system will probably benefit longitudinal evaluation of facial weakness greatly.

This study provides an overview of the clinical spectrum of facial weakness and its relation to other disease characteristics. The task of the developing of an objective and accurate outcome measure for facial weakness is highly challenging and requires more research. The simple four point scale we introduced to grade the severity of facial weakness, enables correlation of facial weakness to disease characteristics, but is not suited as a clinical outcome measure for longitudinal studies.

Author contributions TGJL, Radboud University Medical Center, Nijmegen, the Netherlands: acquisition of data, analysis and interpretation of data, drafting of manuscript and tables/figures. $\mathrm{CGCH}$ : study concept and design, revision of manuscript. SCCV: Acquisition and interpretation of data, drafting of manuscript and tables/figures. CHGB: acquisition of data, revision of the manuscript. SK: acquisition of data, revision of the manuscript. GWAMP: acquisition of data, revision of the manuscript. NCV: revision of the manuscript. TJJM: revision of the manuscript. BGME: study concept and design, revision of the manuscript. KM: study concept and design, acquisition of data, analysis and interpretation of data, drafting of manuscript and tables/ figures. JMS, University of Kansas Medical Center, Kansas City, Kansas, USA: revision of the manuscript.

Funding Not applicable.

Availability of data and material The data that support the findings of this study are available from the corresponding author upon reasonable request.

Code availability Not applicable. 


\section{Compliance with ethical standards}

Conflicts of interest The authors report no conflicts of interest.

Ethics approval This study was conducted according to the principles of the Declaration of Helsinki (version October 2013) and in accordance with the Medical Research Involving Human Subjects Act (WMO). The study protocol was approved by the regional medical ethics committee (CMO Arnhem-Nijmegen). Prior to inclusion, all participants signed informed consent and if applicable a consent-todisclose form.

Open Access This article is licensed under a Creative Commons Attribution 4.0 International License, which permits use, sharing, adaptation, distribution and reproduction in any medium or format, as long as you give appropriate credit to the original author(s) and the source, provide a link to the Creative Commons licence, and indicate if changes were made. The images or other third party material in this article are included in the article's Creative Commons licence, unless indicated otherwise in a credit line to the material. If material is not included in the article's Creative Commons licence and your intended use is not permitted by statutory regulation or exceeds the permitted use, you will need to obtain permission directly from the copyright holder. To view a copy of this licence, visit http://creativecommons.org/licenses/by/4.0/.

\section{References}

1. Mul K, Lassche S, Voermans NC, Padberg GW, Horlings CG, van Engelen BG (2016) What's in a name? The clinical features of facioscapulohumeral muscular dystrophy. Pract Neurol 16:201-207

2. Mul K, Berggren KN, Sills MY et al (2019) Effects of weakness of orofacial muscles on swallowing and communication in FSHD. Neurology 92:e957-e963

3. Padberg GW (1982) Facioscapulohumeral disease. University of Leiden, Leiden

4. Chung CS, Morton NE (1959) Discrimination of genetic entities in muscular dystrophy. Am J Hum Genet 11:339-359

5. Mul K, Voermans NC, Lemmers R et al (2018) Phenotype-genotype relations in facioscapulohumeral muscular dystrophy type 1. Clin Genet 94:521-527
6. Lamperti C, Fabbri G, Vercelli L et al (2010) A standardized clinical evaluation of patients affected by facioscapulohumeral muscular dystrophy: The FSHD clinical score. Muscle Nerve 42:213-217

7. Ricci E, Galluzzi G, Deidda G et al (1999) Progress in the molecular diagnosis of facioscapulohumeral muscular dystrophy and correlation between the number of $\mathrm{KpnI}$ repeats at the 4q35 locus and clinical phenotype. Ann Neurol 45:751-757

8. Butz M, Koch MC, Muller-Felber W, Lemmers RJ, van der Maarel SM, Schreiber H (2003) Facioscapulohumeral muscular dystrophy. Phenotype-genotype correlation in patients with borderline D4Z4 repeat numbers. J Neurol 250:932-937

9. Felice KJ, North WA, Moore SA, Mathews KD (2000) FSH dystrophy $4 \mathrm{q} 35$ deletion in patients presenting with facial-sparing scapular myopathy. Neurology 54:1927-1931

10. He JJ, Lin XD, Lin F et al (2018) Clinical and genetic features of patients with facial-sparing facioscapulohumeral muscular dystrophy. Eur J Neurol 25:356-364

11. Ricci G, Cammish P, Siciliano G, Tupler R, Lochmuller H, Evangelista T (2019) Phenotype may predict the clinical course of facioscapolohumeral muscular dystrophy. Muscle Nerve 59:711-713

12. Krasnianski M, Eger K, Neudecker S, Jakubiczka S, Zierz S (2003) Atypical phenotypes in patients with facioscapulohumeral muscular dystrophy 4q35 deletion. Arch Neurol 60:1421-1425

13. Mostacciuolo ML, Pastorello E, Vazza G et al (2009) Facioscapulohumeral muscular dystrophy: epidemiological and molecular study in a north-east Italian population sample. Clin Genet 75:550-555

14. Felice KJ, Moore SA (2001) Unusual clinical presentations in patients harboring the facioscapulohumeral dystrophy $4 \mathrm{q} 35$ deletion. Muscle Nerve 24:352-356

15. Hassan A, Jones LK Jr, Milone M, Kumar N (2012) Focal and other unusual presentations of facioscapulohumeral muscular dystrophy. Muscle Nerve 46:421-425

16. Pastorello E, Cao M, Trevisan CP (2012) Atypical onset in a series of 122 cases with FacioScapuloHumeral Muscular Dystrophy. Clin Neurol Neurosurg 114:230-234

17. Felice KJ, Whitaker CH (2005) The clinical features of Facioscapulohumeral Muscular Dystrophy associated with borderline $(\geq 35$ kb) 4q35 EcoRI fragments. J Clin Neuromuscul Dis 6:119-126

18. Linstrom CJ (2002) Objective facial motion analysis in patients with facial nerve dysfunction. Laryngoscope 112:1129-1147 